\title{
A potential misdiagnosis in the COVID-19 pandemic era: superimposed thrombosis or recurrence
}

\author{
Arezou Zoroufian ${ }^{1}$, Reza Mohseni badalabadi ${ }^{1}$, Mehrdad Mahalleh $^{1}$, Seyyed Mojaba \\ Ghorashi $^{1}$, Sharam Momtahen ${ }^{1}$, and Negar Omidi ${ }^{1}$ \\ ${ }^{1}$ Tehran Heart Center
}

November 9, 2020

\begin{abstract}
The clinical manifestation of coronavirus disease 2019 (COVID-19) overlap with other disorders especially cardiovascular diseases (CVD). We report a 58 years old female presented with syncope episodes and dyspnea on exertion with the left atrial mass, candidate for surgical removal and mitral valve replacement. Nearly 3 months later, she developed dyspnea, fever, and a sore throat cause to admit her with suspected COVID-19. During diagnostic evaluation, a larger LA mass detected that seemed to be COVID-19 induced organized thrombus with prosthetic mitral valve malfunction. the mass resected and immunohistochemistry revealed liposarcoma. The unusual early recurrence of liposarcoma and misdiagnosis with COVID-19 induced thrombosis are the hallmark of the present case.
\end{abstract}

A potential misdiagnosis in the COVID-19 pandemic era: superimposed thrombosis or recurrence

Authors:

Arezou Zoroufian ${ }^{1}$

arezou.zoroufian@yahoo.com

Reza Mohseni-Badalabadi ${ }^{1}$

mohsenihr@yahoo.com

Mehrdad Mahalleh ${ }^{1}$

mahallehm@yahoo.com

Seyyed Mojaba Ghorashi ${ }^{1}$

mojtaba.ghorashi8@gmail.com

Sharam Momtahen ${ }^{1}$

Parichehr.pasbakhsh@gmail.com

Negar Omidi ${ }^{1}$

negar.omidi@gmail.com

1. Teharn Heart Center, Tehran University of Medical sciences, Tehran, Iran

Corresponding author:

Negar Omidi 
Tehran Heart Center, Tehran University of Medical Sciences

Tel: +982188029600

Fax: +982188029731

E-mail: negar.omidi@gmail.com

Conflicts of interest: none declared

Funding: none

Data Availability Statements: The data underlying this article will be shared on reasonable request to the corresponding author.

Clinical trial registration: N/A

\begin{abstract}
The clinical manifestation of coronavirus disease 2019 (COVID-19) overlap with other disorders especially cardiovascular diseases (CVD). We report a 58 years old female presented with syncope episodes and dyspnea on exertion with the left atrial mass, candidate for surgical removal and mitral valve replacement. Nearly 3 months later, she developed dyspnea, fever, and a sore throat cause to admit her with suspected COVID-19. During diagnostic evaluation, a larger LA mass detected that seemed to be COVID-19 induced organized thrombus with prosthetic mitral valve malfunction. the mass resected and immunohistochemistry revealed liposarcoma. The unusual early recurrence of liposarcoma and misdiagnosis with COVID-19 induced thrombosis are the hallmark of the present case.
\end{abstract}

Key words: liposarcoma, COVID-19, thrombosis

\title{
Introduction
}

The recent pandemic of COVID-19 has become the most challenging issue worldwide, resulting in more than 40 million identified cases with more than 1 million death. A similar presentation between COVID-19 and CVD has hindered in the diagnosis and management of CVD, especially in patients with prosthetic heart valves. ${ }^{1-3}$ Primary cardiac sarcomas are rare, aggressive, and most lethal. The autopsy incidence of the primary cardiac neoplasm is extremely low about $0.2 \%$ of all cardiovascular surgical cases. Of the $25 \%$ of these tumors that are classified as being malignant, cardiac sarcomas make up $95 \%$ of cases. ${ }^{4-6}$

Depending on the location of the tumor and the range of infiltration into the adherent tissue, a cardiac tumor can cause symptoms which are generally nonspecific. The diagnosis is reached from the clinical history and multimodality imaging. Surgical removal and radiotherapy are used as a basis for local restriction, accompanied by chemotherapy for systemic disease. ${ }^{4,7}$ We herein describe a middle-aged female with a Left atrial mass which was suspected as myxoma before surgery. Another LA mass showed up about 3 months after the complete surgical resection. The new mass was suspected COVID-19 infection-induced thrombosis. It was again resected and diagnosed as Dedifferentiated Liposarcoma (DDLPS) by postoperative pathological examination. This case highlights the fact that a precise diagnosis is crucial as the treatment approach and the accompanying risks differ in different conditions.

\section{Methods}

The patient provided written informed consent and the investigation conformed to the principles outlined in the Declaration of Helsinki. Approval for the study protocol was obtained from the institutional ethics committee of THC.

\section{Case Presentation}


A 58 years old female presented with a 2-month history of dyspnea on exertion (DOE) and two episodes of syncope was admitted on January $20^{\text {th }}, 2020$. The transthoracic echocardiography (TTE) showed a welldefined heterogeneous large round mobile mass with attachment to the atrial side of anterior mitral valve leaflet (AMVL) $\left(29^{*} 20 \mathrm{~mm}\right)$ suggestive of an atypical myxoma (Figure 1). In addition, the Ejection Fraction (EF) of $55 \%$, severe mitral stenosis, severe mitral regurgitation, mild to moderate tricuspid regurgitation, and pulmonary arterial systolic pressure (PASP) of $42 \mathrm{mmHg}$ were reported. The coronary angiography revealed normal epicardial coronary arteries. The brain CT and neurologic evaluation results were unremarkable. The patient became a candidate for surgical mass excision. On January $24^{\text {th }}$, the LA mass was removed completely $(2 * 2.5 \mathrm{~cm})$ and the mitral valve was replaced with the St. Jude Medical prosthesis due to the involvement of AMVL. The histopathological examination revealed diffuse fibrosis of mitral valve and cellular pleomorphism and high mitotic activity in the removed cardiac tumor. The evaluation of the surgical margin was not possible. Postoperative echocardiography revealed that the prosthetic valve had good leaflet motion with an acceptable gradient. The EF of $45 \%$, Mild TR, and PASP of $28 \mathrm{mmHg}$ were also reported. No residual mass was detected. The patient was discharged thereafter on February $3^{\text {rd }}$.

Seventy-nine days later on April 22 ${ }^{\text {nd }}, 2020$, the patient was readmitted with a 1-week history of dyspnea, palpitation, fever (body temperature of $38{ }^{\circ} \mathrm{C}$ ), and sore throat. The time in therapeutic range assessment revealed that the international normalized ratio (INR) was acceptable during the mentioned period. In the emergency department, the TTE revealed fixation of one of leaflet of the prosthetic mitral valve with a mean pressure gradient of $12 \mathrm{mmHg}$, LVEF of $50 \%$, moderate to severe TR and PASP of $63 \mathrm{mmHg}$. The mitral valve fixation was evident in the fluoroscopy. Laboratory examination demonstrated the high sensitive C-reactive protein (hs-CRP) of $9.9 \mathrm{mg} / \mathrm{dl}$ and white blood cell of $7100 / \mu \mathrm{L}$ with lymphocyte percentage of $18.8 \%$. In the COVID-19 pandemic era, the focus was diverted to the thrombotic event of the prosthetic valve in the context of coronavirus infection and a spiral chest computed tomography (CT) scan performed to exclude the lung involvement. Although, no evidence of COVID-19 was detected in chest CT except mild pleural effusion, empiric antiviral treatment with hydroxychloroquine and azithromycin was initiated with respect to symptoms and high hs-CRP. The blood culture examination result was negative. The transesophageal echocardiography (TEE) revealed a very large non-homogenous LA mass $(4 * 4 \mathrm{~cm})$ on the lateral LA wall that seemed to be an organized thrombus and restricted mitral valve leaflet motion (Figure 2). The reverse transcription-polymerase chain reaction test was negative for COVID-19. The patient was a candidate for surgical removal of the mass which was thought to be a thrombus $\left(4^{*} 4 \mathrm{~cm}\right)$. Surgical resection of the mass was done on $27^{\text {th }}$ April and the gross appearance was not compatible with thrombus or pannus. The postoperative echocardiography on May $2^{\text {nd }}$ revealed LVEF of $40 \%$, good leaflet motion of the prosthetic mitral valve. No visible residual mass was detected. The operative specimen was sent for pathological assessment. The Immunohistochemical stain raised the diagnosis of liposarcoma. The primary origin of the tumor approved, hence, the patient referred to the oncology department.

\section{Discussion}

DDLPS is a non-lipomatous malignancy with high cellularity, considerably more aggressive phenotype rising the risk of systemic metastases, and local recurrence. ${ }^{8}$ In this case, the patient experienced an in-situ recurrence of the sarcoma within only 3 months after the complete resection which was unusual.

The 5-year survival rate for malignant cardiac tumors following treatment was about $30 \%$. the recurrence rate for liposarcoma was reported in about $40 \%$ of the cases even up to 14 years after the initial surgical resection. ${ }^{4,8}$ In primary cardiac DDLP, most cases are younger than 45 years old and no sex predilection has been seen. The clinical features are generally related to the site of the tumor and the extent of infiltration. ${ }^{9}{ }^{10}$ According to the data available, the most frequent symptoms in malignant cardiac tumors are dyspnea and chest pain, moreover, fever is also reported with a prevalence of $9 \% .{ }^{5,11}$ In the present case, the questionable finding was a LA mass that developed within 79 days after primary surgical resection and resulted in dyspnea. It was crucial to differentiate valvular thrombus from other possibilities such as a remnant or recurrent tumor. The absence of constitutional symptoms was weighting against the recurrent malignant tumor. 
On condition that the patient has satisfactory performance status, surgical resection of the intracavitary mass should be done. Even though cardiac tumors seem to be a challenge for cardiac surgeons, complete surgical resection combined with adjuvant chemotherapy and/or local radiotherapy is required to lower the risk of local recurrence and systemic metastasis. ${ }^{4}{ }^{11}$ It should be pointed out that due to heart failure which is possible in the early course of the left-sided mass, neoadjuvant chemotherapy is generally contraindicated. ${ }^{12}$ This case was notable for the short interval to recurrence. Completeness of resection is a helpful feature in disease-free survival and without adjuvant chemotherapy recurrence of cardiac sarcomas increases. Recurrence of primary cardiac sarcomas is a common phenomenon and close surveillance with an oncologist is essential.

\section{Conclusions}

due to the concerns around the COVID-19 pandemic era, misdiagnosis can occur in cardiovascular disorders with COVID-19 infection. In this case, the attempt to be alert in the diagnosis and empiric treatment of COVID-19, resulted in the misdiagnosis. Even though the CT scan and RT-PCR results showed no evidence in the favor of COVID-19, the symptoms of the patient and the TEE results were thought to be related to the thrombosis of the prosthetic valve caused by the infection. Although we need to be vigilant in the diagnosis of COVID-19, we should not forget about the other possible disorders.

\section{References}

1. Omidi N, Forouzannia SK, Poorhosseini H, Tafti SHA, Salehbeigi S and Lotfi-Tokaldany M. Prosthetic heart valves and the COVID-19 pandemic era: What should we be concerned about? Journal of Cardiac Surgery . 2020;35:2500-2505.

2. Shafi AM, Shaikh SA, Shirke MM, Iddawela S and Harky A. Cardiac manifestations in COVID-19 patients-A systematic review. Journal of cardiac surgery . 2020;35:1988-2008.

3. Bansal M. Cardiovascular disease and COVID-19. Diabetes $\&$ Metabolic Syndrome: Clinical Research $\&$ Reviews . 2020.

4. Hoffmeier A, Sindermann JR, Scheld HH and Martens S. Cardiac tumors-diagnosis and surgical treatment. Dtsch Arztebl Int . 2014;111:205-211.

5. Ramlawi B, Leja MJ, Abu Saleh WK, Al Jabbari O, Benjamin R, Ravi V, Shapira OM, Blackmon SH, Bruckner BA and Reardon MJ. Surgical Treatment of Primary Cardiac Sarcomas: Review of a SingleInstitution Experience.Ann Thorac Surg . 2016;101:698-702.

6. Look Hong NJ, Pandalai PK, Hornick JL, Shekar PS, Harmon DC, Chen YL, Butrynski JE, Baldini EH and Raut CP. Cardiac angiosarcoma management and outcomes: 20-year single-institution experience. Ann Surg Oncol . 2012;19:2707-2715.

7. Conyers R, Young S and Thomas DM. Liposarcoma: molecular genetics and therapeutics. Sarcoma . 2011;2011:483154.

8. Steger CM. Primary liposarcoma of the heart. Case Reports . 2011;2011:bcr0320114013.

9. Ghadimi MP, Al-Zaid T, Madewell J, Peng T, Colombo C, Hoffman A, Creighton CJ, Zhang Y, Zhang A, Lazar AJ, Pollock RE and Lev D. Diagnosis, management, and outcome of patients with dedifferentiated liposarcoma systemic metastasis. Ann Surg Oncol . 2011;18:3762-3770.

10. Travis WD, Brambilla E, Burke AP, Marx A and Nicholson AG. Introduction to The 2015 World Health Organization Classification of Tumors of the Lung, Pleura, Thymus, and Heart. J Thorac Oncol . 2015;10:1240-1242.

11. Simpson L, Kumar SK, Okuno SH, Schaff HV, Porrata LF, Buckner JC and Moynihan TJ. Malignant primary cardiac tumors: review of a single institution experience. Cancer . 2008;112:2440-2446. 
12. Blackmon SH and Reardon MJ. Surgical treatment of primary cardiac sarcomas. Texas Heart Institute journal . 2009;36:451-452.

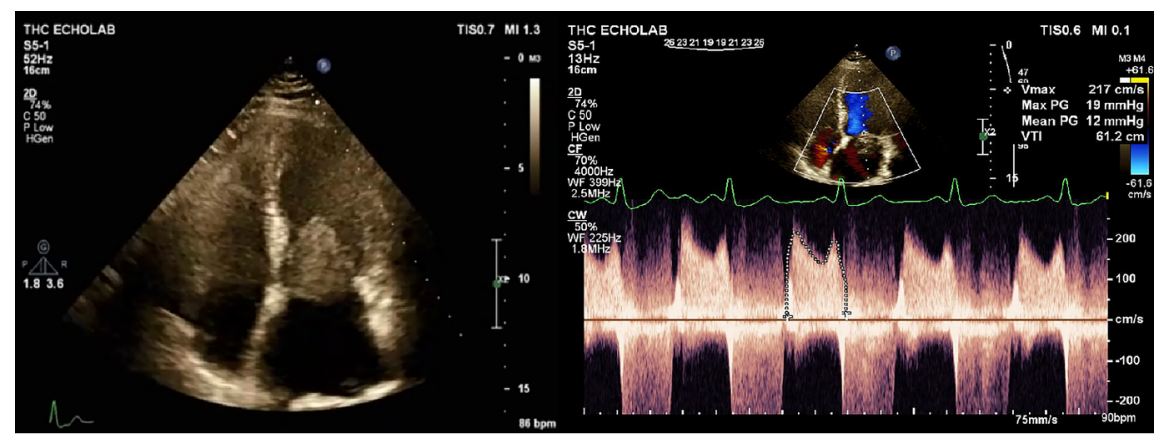

Figure 1. large round mobile mass attached to tip of anterior mitral valve leaflet $(29 * 20 \mathrm{~mm})$ protrude to left ventricule producing significant functional mitral stenosis (apical 4-chamber view in transthoracic echocardiography).

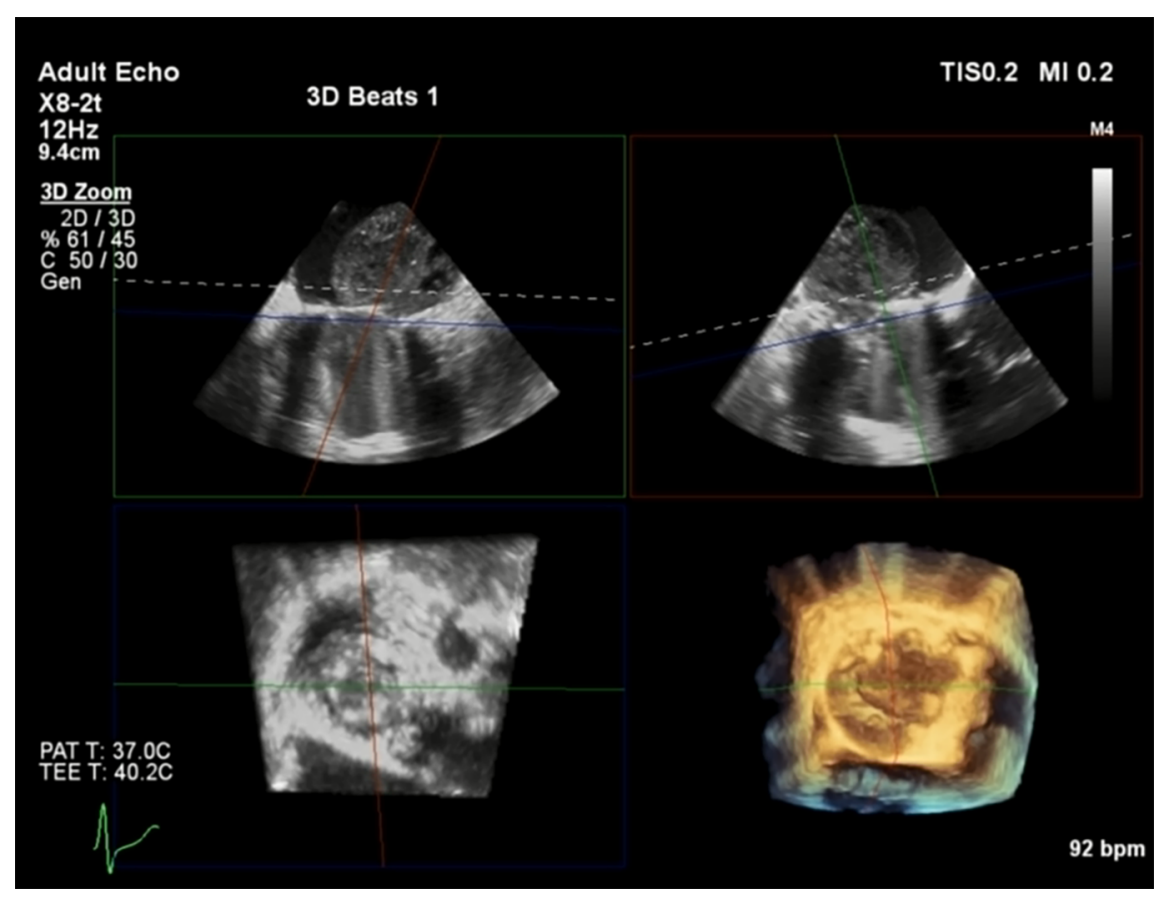

Figure 2. large non-homogenous fixed mass $(4 * 4 \mathrm{~cm})$ on lateral left atrial wall with restricted mitral valve motion (mid-esophageal view and three-dimensional image in transesophageal echocardiography). 

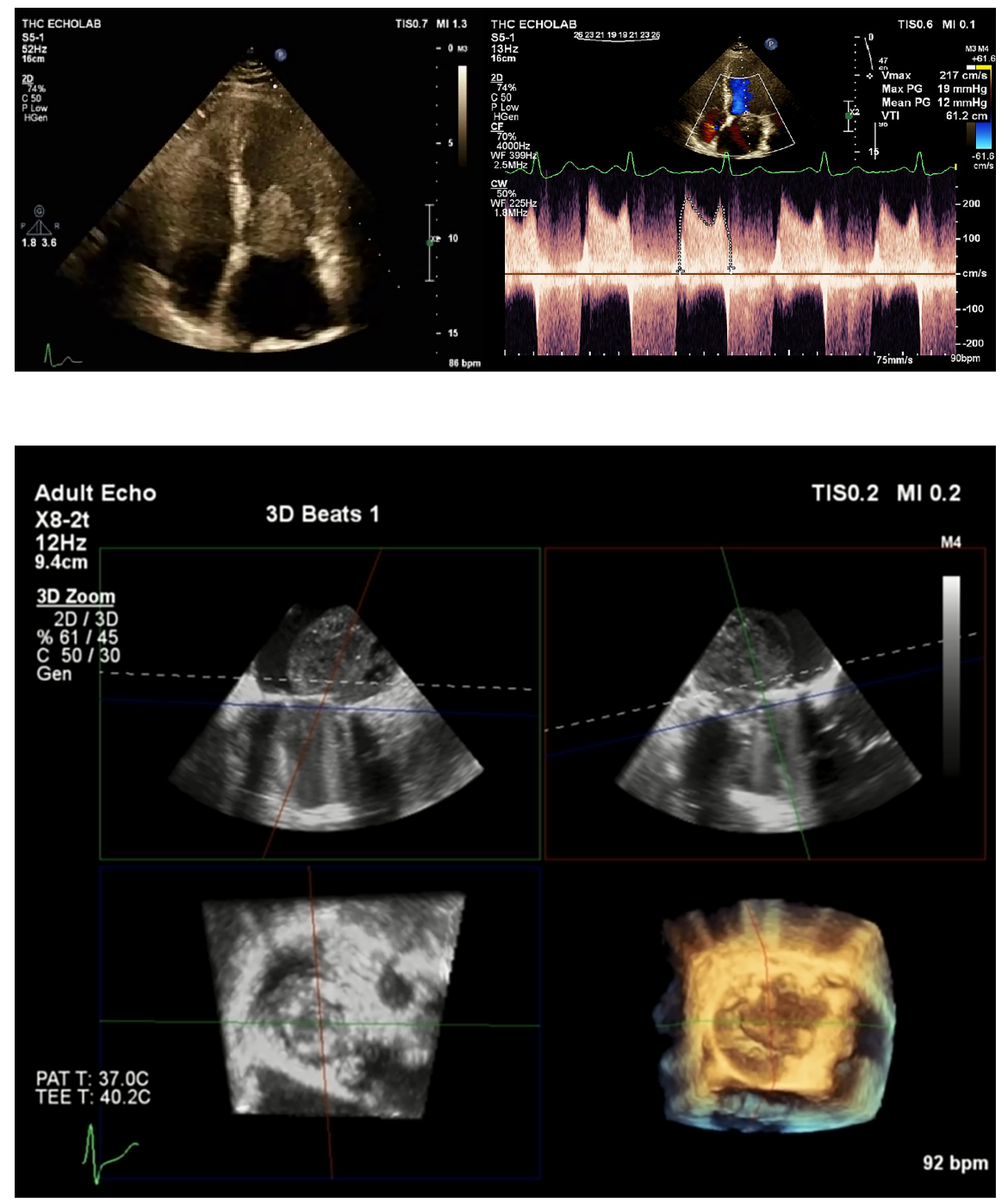